\title{
Comparison of Image Analysis Methods in Skin Temperature Measurements during Physical Exercise
}

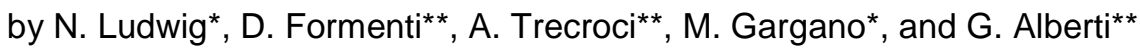 \\ *Department of Physics, State University of Milan, Italy. nicola.ludwig@unimi.it \\ **Department of Biomedical Sciences for Health, State University of Milan, Italy. damiano.formenti@unimi.it
}

\begin{abstract}
The analysis of thermographic images in biomedical application is usually performed by different methods of averaging the values of pixel temperature inside a Region of Interest (ROI). We used a new method, based on maximal temperatures inside a ROI (Tmax), to evaluate temperature modifications during physical exercise in trained or untrained subjects. In this paper we analysed squat exercise effects on the thighs of 6 active subjects. Data were compared with those obtained with traditional method based on the average over an area in the anatomical part under investigation. The values of temperature measured before, during and after the exercise showed the same pattern with both methods. A slightly decrease in temperature during the exercise was followed by an increase during the recovery time. Bland and Altman statistical analysis confirmed the good agreement between the new method and the one based on the average considered as the gold standard. In this study we put in evidence the features of this analysis method specifically in human application.
\end{abstract}

\section{Introduction}

In the last years, Infrared Thermography [IRT] in the biomedical sciences progressively turned into a widespread technique to detect variations in skin temperature depending on physical activity. Several authors measured skin temperatures during exercise in different parts of the human body: dorsal hand during office work [1], total body during running exercise [1], dorsal hand during cycling exercise [2], seven area on trunk during breathing exercise [3] and calves during resistance exercise [4]. These studies focused on the skin temperature response to physical exercise in different categories of subjects such as trained/untrained and old/young.

Skin temperature depends on anatomical factors such as the presence of hair or other irregularities on the surface of the skin but especially on the presence of subcutaneous fat or muscular tissue, as well as a physiological factor concerning the thermoregulation mechanism through blood flow in the vascular tissues. Vasodilation and vasoconstriction in physical exercise are, effectively, the main mechanism for heat dissipation [5]. All these factors can influence the skin temperature and its spatial distribution. Thus, it is frequent to find a non-Gaussian thermal distribution around mean temperature value inside the selected ROI. Figure 1 shows the thighs of one subject with such a typical thermal pattern.

The thermal images analysis is usually performed by dedicated software that allows to select a Region of Interest (ROI) on the body surface and calculates the mean value and its standard deviation over all the pixels inside such region. For physiological purpose in exercise-associated skin temperature investigations, as already proposed by [4], it is important to specify the method used to calculate the temperature value representative of the area of interest. One method based on maximal temperature was used in order to evaluate the skin temperature of calves during and after exercise [4], and a method comparison study was recently performed at rest condition [7].

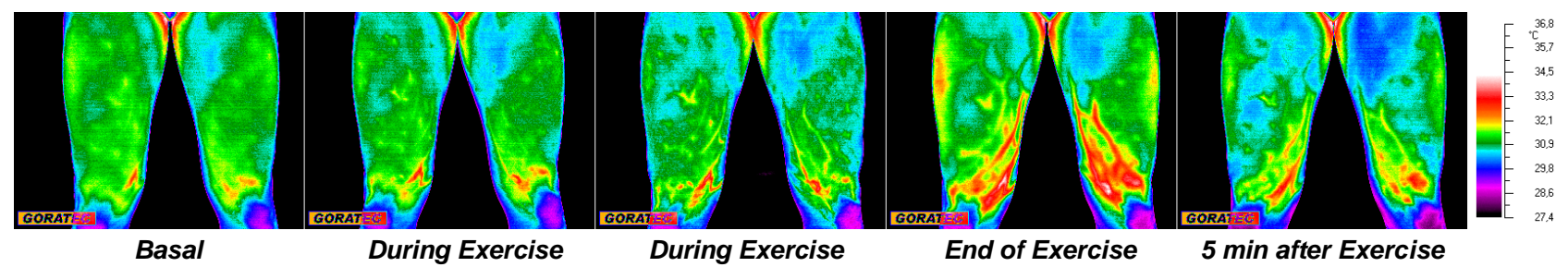

Fig. 1. Temperature distribution on thighs of one subject at different stages of the trial.

The aim of this study was to compare the Troi method [widely used in literature] with the Tmax method [7] in the evaluation of skin temperature during exercise. Thermal images on the quadriceps of active subjects performing squat exercise with overload were recorded, and subsequently analysed using Troi and Tmax methods. We hypothesized that 
the differences between Troi and Tmax method already assessed in basal conditions [7] would be found again during exercise conditions.

\section{Methods}

Six male active subjects volunteered to take part in the study. They belonged to the student population of the Faculty of Exercise Science of the State University of Milan. Their mean age and body mass index were $25.8 \pm 1.8$ years, $22.3 \pm 2.5 \mathrm{~kg} / \mathrm{m}^{2}$, respectively. They were habitually physical active but none of them were familiar with specific resistance exercise. All participants were non smokers, and without cardiovascular or pulmonary diseases. They had not assumed drugs or medications with a potential effect on cardiovascular and thermoregulatory functions during the two months prior the tests. The Ethical Committee of the State University of Milan approved this study. After a thorough explanation of the protocol that was going to be used, the subjects accepted informed written consent to participate in this study.

The subjects were instructed to refrain from strenuous physical activity the day before the trials and abstained, as well, from assuming alcoholic or caffeine-containing products for a 4-h period before to the start of the experiment.

In a preliminary session one week before the experiment, a one-repetition maximum squat test (1-RM) was performed by each subject for determining the individual's maximum strength at parallel squat movement [8].

In laboratory conditions, with environmental temperature and relative humidity controlled $\left[22-23^{\circ} \mathrm{C}, 50 \pm 5 \%\right]$, they carried on one set of repetitions until exhaustion of parallel squat movement with a load of $50 \%$ of 1 Maximal Repetition [1-RM]. Thermal images were recorded every $20 \mathrm{~s}$ during the $2 \mathrm{~min}$ of pre-exercise to determinate basal temperature, during the exercise and the recovery-time lasting $10 \mathrm{~min}$, and were subsequently analyzed by using the two different methods (Troi and Tmax).

An operator selected a ROI per each thigh, and finally the temperature value for Troi method was calculated as arithmetic mean over the temperature value of all the pixels inside this ROI.

The Tmax temperature is a value obtained by using the values of temperature of the five hottest pixel inside all the anatomical part under consideration. Tmax method was calculated following the procedure already proposed $[3,6]$. Such procedure was previously used for calf temperature [6], while in this study it was used to obtain a reliable temperature of the thigh surface. One region of interest including all the muscles involved in exercise was selected for each thigh. Then the software selected automatically the five hottest pixels inside this area. These pixels were selected imposing a minimum distance of 5 pixels among each other. Then, temperature value was obtained by averaging over an area of $5 \times 5$ pixels around the hottest five. In this way the result represented an overall amount of 125 pixels on each thigh. In this way, for every thermal image, each subject was represented by a temperature value calculated using the same number of pixels, those representative of cutaneous area essential for heat dissipation.

Troi and Tmax values were calculated at basal, end of exercise, and $5 \mathrm{~min}$ after exercise and were considered in statistical analysis. First, after the confirmation of the normal distribution of the variables [Kolgomorv-Smirnov normality test] left and right thigh temperatures were averaged, thus obtaining only one temperature value per subject: to compare the two methods, a paired $t$ test was used at each stage of the protocol. Second, to verify the strength of the relationship between the two methods, Pearson correlation coefficient was calculated considering right and left thigh separately, for all the three stages of the trial [basal, end of exercise, $5 \mathrm{~min}$ after exercise]. Third, to determine the validity of the Tmax against the Troi [identified as gold standard], the Bland and Altman plot was used considering right and left thigh separately [9]. A significance level at $\alpha=0.05$ was established for statistical analysis by using Graphpad Prism 5.01 software. Data are presented as mean \pm standard deviation.

\section{Results}

Figure 1 shows thermal images before [basal shot], during [second and third shot], at the end of the exercise [fourth shot] and at $5 \mathrm{~min}$ after the end of the exercise [fifth shot]. From the third shot it is possible to notice that a characteristic pattern of the superficial vessels became visible on the quadriceps muscle. (After comparing several images) we chose 3 phases as those representative of the trial basal, end of exercise, and recovery. All the subjects showed a decrease in thighs skin temperature from basal to end of exercise condition, recorded in both methods (Figure 2 and 3 ) as observed by others authors $[3,9]$. Skin temperature changes during the recovery time -5 min after exerciseis less evident, in fact uniquely in few subjects we observed a rise in their skin temperature, with the result that variations occur only during the exercise. 
Skin temperature evolution by using Troi data

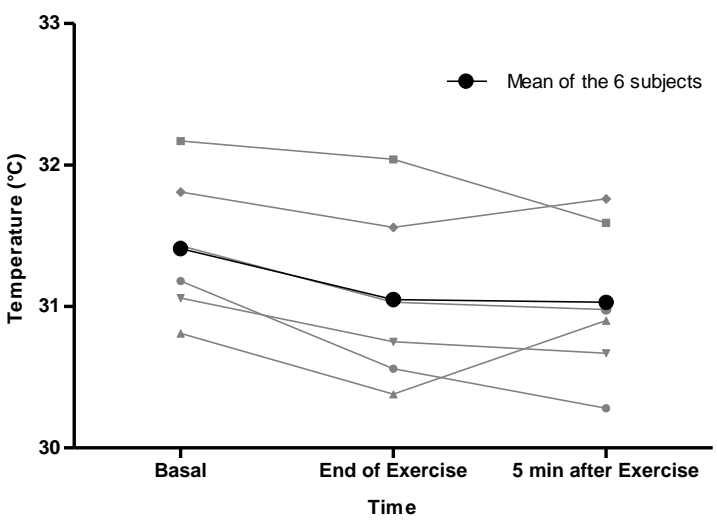

Skin temperature evolution by using Tmax data

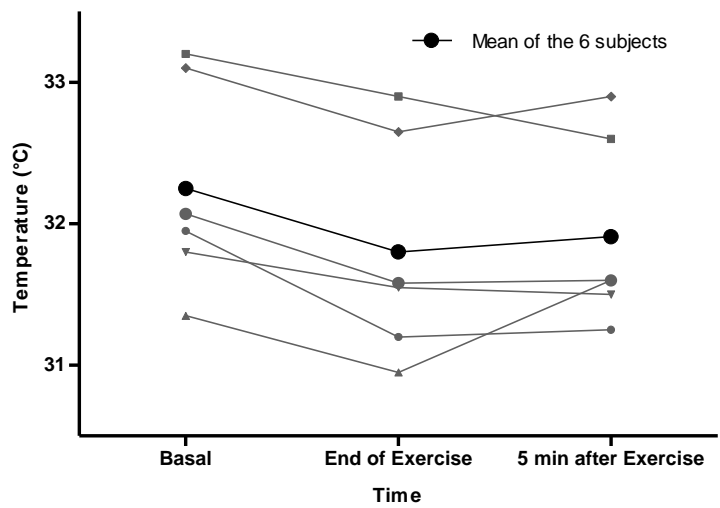

Fig. 2. Skin temperature evolution by using Troi. Grey dots are the thighs temperature values of each subject. Black dots are the mean of the 6 subjects.

Fig. 3. Skin temperature evolution by using Tmax. Grey dots are the thighs temperature values of each subject. Black dots are the mean of the 6 subjects.

The thigh temperature values calculated by using Troi were significantly lower compared to the calculation by using Tmax at each stage of the trial $[p<0.05]$.

Figure 4 shows the high correlation between Troi method and Tmax method $(R=0.94, p<0.05)$, thus demonstrating their strengthening relationship, also in those areas characterized by non-homogeneous skin temperature during strenuous exercise.

The Bland and Altman analysis of validity as presented in Figure 5 demonstrated, as expected, minimal bias $\left[0.8^{\circ} \mathrm{C}\right]$, and relatively low standard deviations. However, a little increase in skin temperature difference for higher values of mean skin temperature is present.

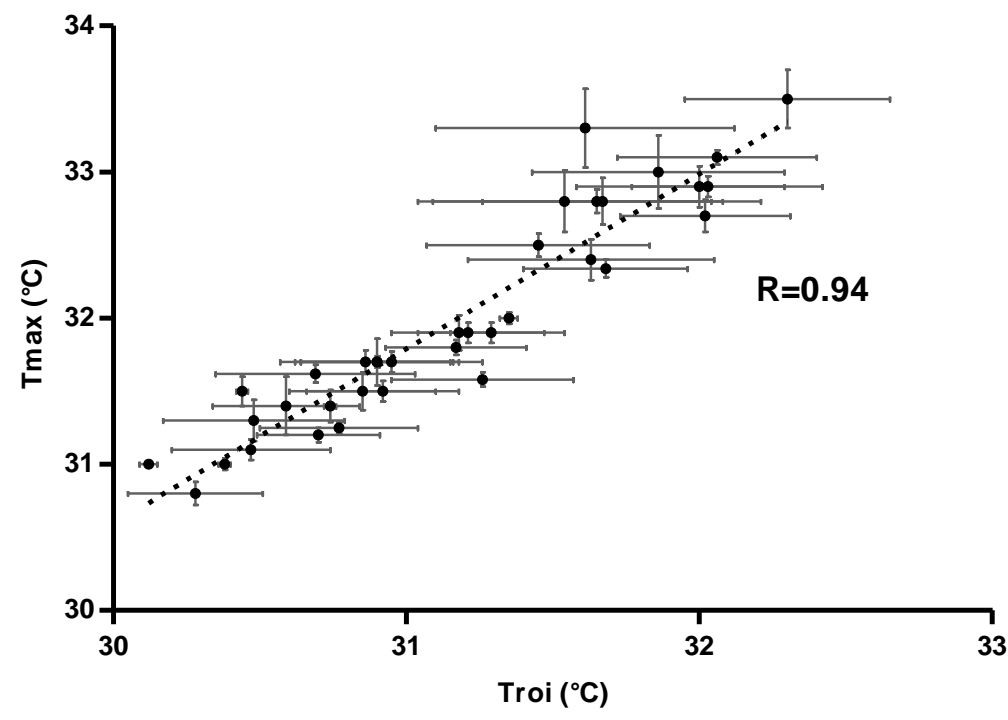

Fig. 4. Relationship between thighs skin temperature values measured by using Troi and Tmax. 


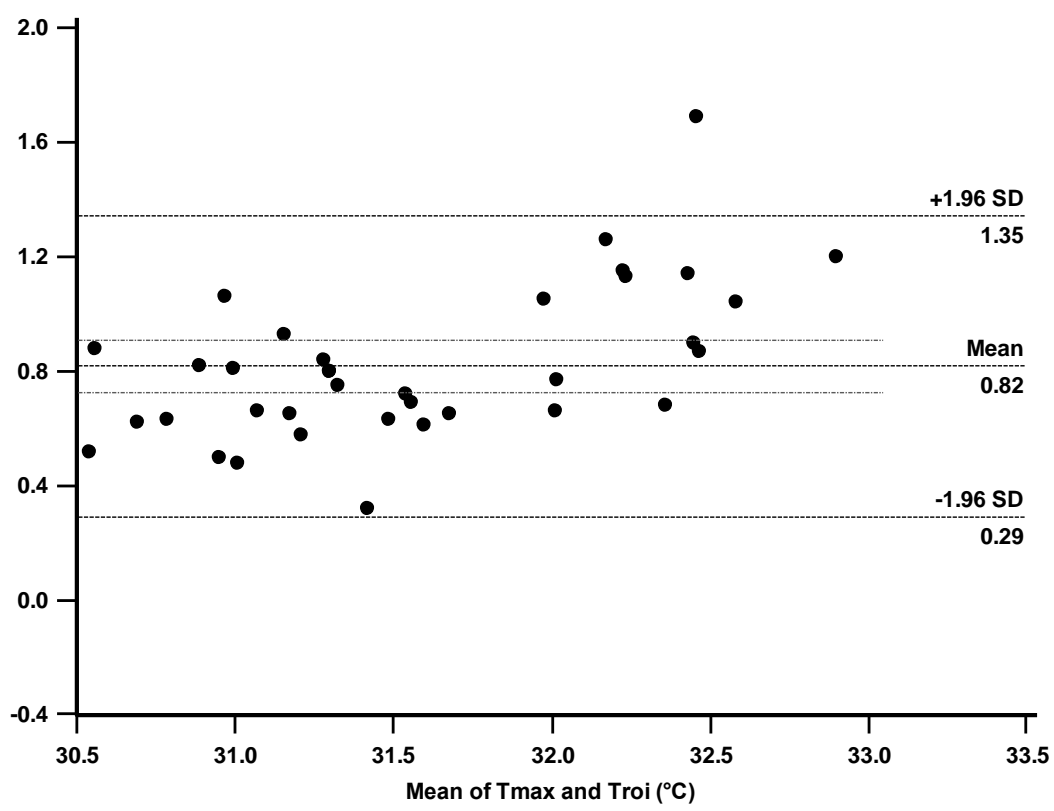

Fig. 5. Bland and Altman plot showing the relationship between mean measured values of Troi and Tmax and their difference.

\section{Discussion}

The purpose of this study was to observe the skin temperature evolution during resistance exercise by using infrared thermography, and to examine the validity of a new method of surface temperature evaluation, particularly useful in skin temperature studies.

Skin temperature evolution during the trial has shown a decrease of some tenth of degree from the start until the end of the exercise, probably due to a different intervention of vasoconstriction and vasodilation [5].

All the subjects showed a decrement in skin temperature: it is clear to notice that Tmax method is more adequate and accurate in detecting skin temperature variations $\left(\Delta \operatorname{Troi}=-0.21 \pm 0.25^{\circ} \mathrm{C} ; \Delta \operatorname{Tmax}=-0.44 \pm 0.10^{\circ} \mathrm{C}\right)$. On the other hand, only a slightly increase in skin temperature after exercise was observed $\left(\Delta \operatorname{Troi}=+0.02 \pm 0.28^{\circ} \mathrm{C} ; \Delta \mathrm{Tmax}=+0.10 \pm 0.10^{\circ} \mathrm{C}\right.$ ). Some subjects raised their temperature whereas some others decreased it during the recovery time. This difference in skin temperature response during the recover can be explained by a predominance of vasoconstriction over vasodilation or vice versa [5,9], or by different physical characteristics (e.g. body mass, fat tissues, metabolism).

For what concern method comparison, the significant difference between Troi and Tmax method at each stage of the trial $(p<0.05)$ was due to the feature of Tmax method in considering pixels with maximal temperature values only [6]. The high value of Pearson correlation coefficient demonstrates the strength of the relationship between the two series of temperature obtained through Troi and Tmax method, as well as the Bland and Altman plot shows their good agreement [8].

Recording of skin temperature in a specific body area in thermoregulation diagnostic field needs to be reliable and repeatable. Every muscle contraction produces physiological changes reflecting on blood flow that influences skin temperature. Skin temperature depends also on anatomical factors, such as the presence of irregularities on the surface and the presence of different kind of subcutaneous tissue (fat tissue and muscular tissue). These factors can influence the skin temperature distribution of a specific body area thus, it is frequent to find non-regular pattern of thermal distribution of pixels inside a ROI. From these considerations, it is clear that the methods based on Troi averaging cannot be used for all skin areas and in all situations. 


\section{Conclusion}

This work contributes to characterize two different methods to calculate skin temperature response to physical exercise by using infrared thermography. The skin temperature changes obtained by Tmax method were compared successfully with the well-established Troi method. Results showed a good agreement between the two methods and a low expected bias. Tmax method demonstrated a higher precision in temperature evaluation in term of standard deviation with respect to Troi. This is due to the small amount of pixels involved in the calculation of the mean itself. We stress that this method does not give the mean temperature over an area but, at least in athlete surveys, allows a good representation of thermal evolution of muscular tissue involved in the exercise. Temperature evolution during the three phases of the exercise agrees with ones already observed in other studies: a decrease during the work phase and a rising during the recovery time.

\section{REFERENCES}

[1] Gold J.E., Cherniack M., Buchholz B., "Infrared thermography for examination of skin temperature in the dorsal hand of office workers". Eur J Appl Physiol, vol. 93, pp. 245-51, 2004.

[2] Zontak A., Sideman S., Verbitsky O., Beyar R., "Dynamic thermography: analysis of hand temperature during exercise". Ann Biomed Eng, vol. 26, pp. 988-93, 1998.

[3] Ludwig N., Gargano M., Formenti D., Bruno D., Ongaro L., Alberti G., "Breathing training characterization by thermal imaging: a case study". Acta Bioeng Biomech, vol. 3, pp. 41-47, 2012.

[4] Formenti D., Ludwig N., Gargano M., Gondola M., Dellerma N., Caumo A., Alberti G., "Thermal Imaging of ExerciseAssociated Skin Temperature Changes in Trained and Untrained Female Subjects". Ann Biomed Eng, vol. 41, pp. 863-871, 2013.

[5] Kellogg D.L., "In vivo mechanisms of cutaneous vasodilation and vasoconstriction in humans during thermoregulatory challenges". J Appl Physiol, vol. 100, pp. 1709-18, 2006.

[6] Ludwig N., Formenti D., Gargano M., Alberti G., "Skin temperature evaluation by Infrared Thermography: Comparison of Image Analysis Methods". Infrared Phys Technol, vol. 62, pp. 1-6, 2014

[7] O'Shea P. "Sports Performance Series: The parallel squat". NSCA J, vol. 7, pp. 4-6, 1985.

[8] Bland J.M., Altman D.G., "Statistical methods for assessing agreement between two methods of clinical measurement". Lancet, vol. 8, pp. 307-10, 1986.

[9] Merla A., Mattei P.A., Di Donato L., Romani G.L., "Thermal imaging of cutaneous temperature modifications in runners during graded exercise". Ann Biomed Eng, vol. 38, pp. 158-63, 2010. 